\title{
HIFAN 1757
}

HEAVY ION FUSION SCIENCE VIRTUAL NATIONAL LABORATORY, 3rd QUARTER 2009 MILESTONE REPORT, Upgrade plasma source configuration and carry out initial experiments. Characterize improvements in focal spot beam intensity

\section{by}

S. Lidia, A. Anders, F.M. Bieniosek, A. Faltens, W. Greenway, J.Y. Jung, T. Katayanagi, B.G. Logan, C.W. Lee, M. Leitner, P. Ni, A. Pekedis, M. J. Regis, P. K. Roy, P. A. Seidl, W. Waldron Lawrence Berkeley National Laboratory, Berkeley, CA 94720, USA

J.J. Barnard, A. Friedman, D. Grote, Lawrence Livermore National Laboratory, Livermore, CA 94550, USA

M. Dorf, E. Gilson

Princeton Plasma Physics Laboratory

Accelerator Fusion Research Division

Ernest Orlando Lawrence Berkeley National Laboratory

University of California

June 2009

This work was supported by the Director, Office of Science, Office of Fusion Energy Sciences, of the U.S. Department of Energy under Contract No. DE-AC02-05CH11231. 


\title{
HEAVY ION FUSION SCIENCE VIRTUAL NATIONAL LABORATORY
}

\author{
3rd QUARTER 2009 \\ MILESTONE REPORT
}

\begin{abstract}
Upgrade plasma source configuration and carry out initial experiments. Characterize improvements in focal spot beam intensity.
\end{abstract}

S. Lidia, A. Anders, J.J. Barnard, F.M. Bieniosek, M. Dorf, A. Faltens, A. Friedman, E. Gilson, W. Greenway, D. Grote, J.Y. Jung, T. Katayanagi, B.G. Logan, C.W. Lee, M. Leitner, P. Ni, A. Pekedis, M. J. Regis, P. K. Roy, P. A. Seidl, W. Waldron, for the HIFS-VNL

June 30, 2009

\section{Introduction}

Simulations suggest that the plasma density must exceed the beam density throughout the drift compression and focusing section in order to inhibit the space charge forces that would limit the spot size and beam intensity on the target. WDM experiments will therefore require plasma densities up to $10^{14} / \mathrm{cm}^{3}$, with the highest density in the last few centimeters before the target. This work was guided by the simulations performed for the FY09 Q1 milestone.

This milestone has been met and we report results of modifications made to the NDCX beamline to improve the longitudinal and radial distribution of the neutralizing plasma in the region near the target plane. In Section 2, we review pertinent simulation results from the FY09 Q1 milestone. Section 3 describes the design, and beam measurements following installation, of a biased, self-supporting metal grid that produces neutralizing electrons from glancing interception of beam ions. Section 4 describes the design and initial testing of a compact Ferro-Electric Plasma Source (FEPS) that will remove the remaining 'exclusion zone' in the neutralizing plasma close to the target plane. Section 5 describes the modification of the beamline to decrease the gap between the FEPS section exit and the final focus solenoid (FFS). Section 6 presents a summary and conclusions. 


\section{Summary of Q1 milestone results}

For the neutralized drift compression to produce the highest possible beam intensity on target, it is necessary that the plasma density exceed the beam density almost everywhere, so that plasma electrons are free to cancel the strong space-charge field that otherwise would inhibit compression to the desired beam density. Among the main factors limiting the final peak intensity on target is the degree to which gaps in the neutralizing plasma are avoided (other factors include energy fluctuations of the beam, source imperfections, imperfections in the tilt voltage waveform, misalignments, and aberrations in the diode and the magnetic optics).

In our FY09 Q1 milestone report, we described how particle-in-cell simulations clarified the effects on the beam that arise from localized regions of incomplete neutralization along the beam path. The beam focal spot size increases and the peak intensity on target decreases when such regions are present. The results showed where additional plasma sources would improve the beam focusing by comparing results obtained with and without plasma in regions where it is presently absent or under-dense. We have found that, in particular, directly introducing plasma into the region immediately upstream of the final-focusing solenoid, and at larger radii within that solenoid, would be beneficial. A deficit of plasma in the region within a few centimeters of the target plane where the beam density is steeply increasing was found to have little impact on the peak intensity on target due partly to the short distance to the target and the mitigating impact of secondary electrons from the target.

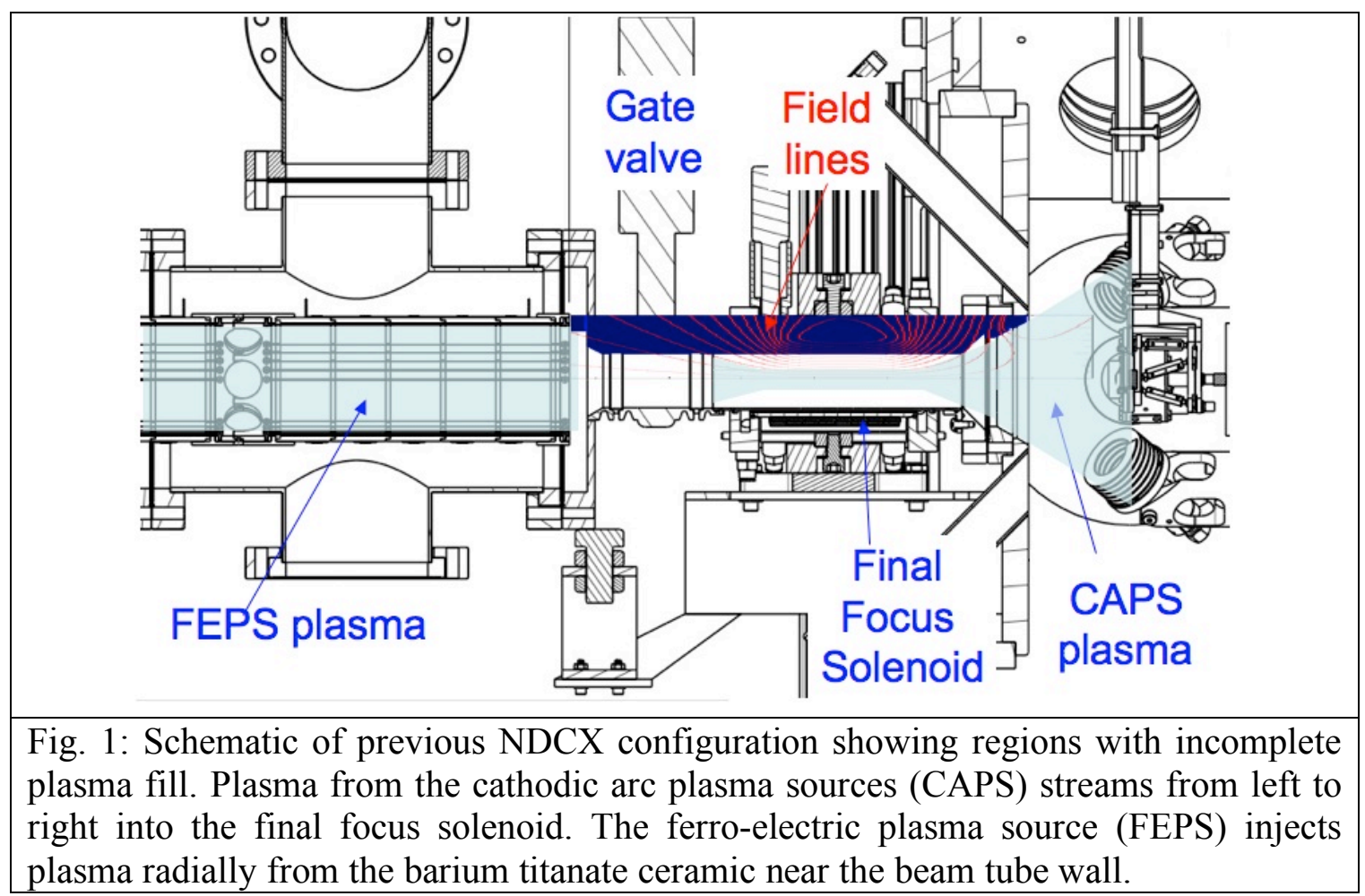


The two significant regions of incomplete plasma fill are shown in Fig. 1. The first region with incomplete plasma fill is the "gap" between the FEPS plasma and the plasma in the final focus solenoid. The second region with incomplete fill is the "exclusion zone" at larger radii within the solenoid, within which plasma is excluded by the combined effect of the magnetic field geometry, the physical structure of the solenoid and associated mounting hardware, and the placement of plasma sources.

The consequences for the ion beam focusing are summarized in Fig. 2. It shows that the gap reduces the peak fluence by an order of magnitude, and the exclusion zone in the final focus solenoid reduces the peak fluence by a factor of two. Both these effects are addressed by experiment modifications described here.

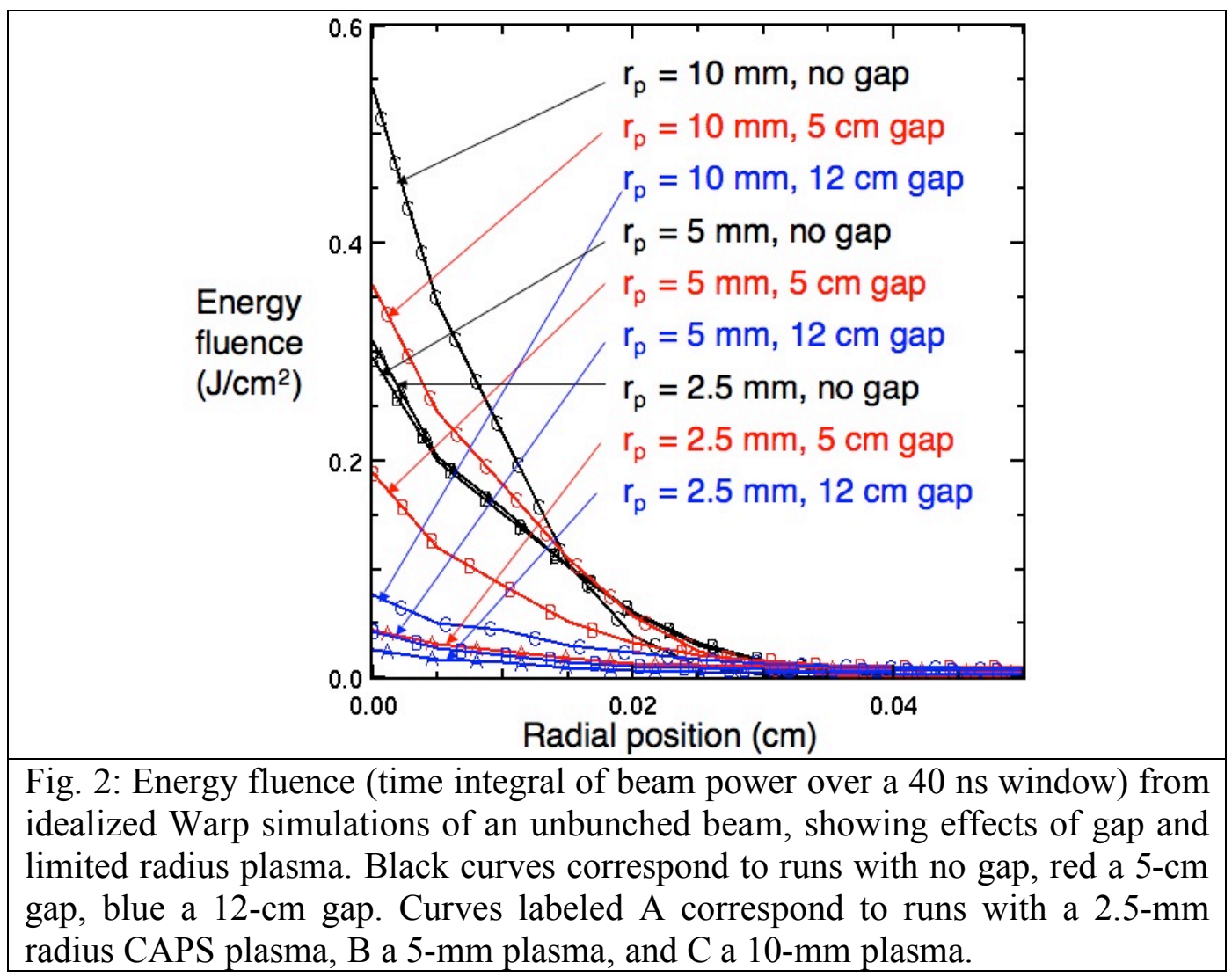

\section{Neutralizing Grid}

Metal grids are a known method of generating electrons due to beam ion impact $[1,2]$. Depending on the angle of incidence, the yield of electrons ranges from $\sim 10$ to $\sim 100$ per primary ion, with the highest values at glancing angles [3]. The simulation results suggested placement of the electron source near the midplane of the 8-Tesla, pulsed final focus solenoid. A generic grid is characterized by a transverse cell size and axial depth. A structurally rigid grid with axial thickness $>1 \mathrm{~mm}$ offers two advantages: one is that it 
allows for more glancing collisions due to axial thickness, and robustness against possible eddy current forces. We have chosen to experiment with a honeycomb shaped hexagonal grid [4] made of non-magnetic steel.

The effective transparency of the grid was estimated using a simplified geometry of the mesh. The model grid was assumed to consist of concentric rings with a finite thickness and length. Model variables include the ring thickness, length, center-to-center spacing, and beam convergence angle.

In WARP simulations, as the main pulse of the beam enters the final focus solenoid, the convergence angle is approximately $15 \mathrm{mrad}$. Estimates of the number of ions lost to head-on collisions (with negligible secondary electron production) with the front face of the grid and of those hitting the mesh plates at a glancing angle indicate an approximate 3:1 ratio of head-on to glancing collisions.

In a solenoid field, the beam will be rotating with a frequency equal to one-half the cyclotron frequency. The fraction of the beam lost due to rotation can be estimated using the similarity with the fraction lost to beam convergence. Finally, with both convergence and rotation playing a role, most of the beam particles that are lost will be at larger radii. Approximately three-quarters of the loss will be in the outer part of the beam beyond half the radius. At the outer radius of $1 \mathrm{~cm}, \sim 20 \%$ of the particles that are lost are due to convergence and $\sim 50 \%$ due to rotation. This has the beneficial effect of producing copious electrons at the radii where the background plasma density has already fallen significantly, while minimizing losses in the core of the beam where the neutralizing plasma resides.

Various grid cell sizes and material thicknesses were considered and the fractional beam loss estimates were calculated. These are tabulated in Table 1. The material highlighted in bold type was chosen as a suitable candidate for the first experiment. The total loss estimated was determined to be tolerable (though it would be preferable to achieve lower loss, while maintaining a high yield of electrons), with a sufficient percentage of glancing collisions for secondary electron production at large radii.

Table 1. Grid materials and loss estimates.

\begin{tabular}{|c|c|c|c|c|c|c|}
\hline \multicolumn{5}{|c|}{ Honeycomb Grid Material } & Fractional Beam Loss \\
\hline Cell Shape & $\begin{array}{c}\text { Cell } \\
\text { diameter } \\
(\mathrm{mil})\end{array}$ & $\begin{array}{c}\text { Foil } \\
\text { thickness } \\
(\mathrm{mil})\end{array}$ & $\begin{array}{c}\text { Axial } \\
\text { depth } \\
(\mathrm{mil})\end{array}$ & $\begin{array}{c}\text { Foil } \\
\text { Material }\end{array}$ & Total & Glancing \\
\hline Hexagonal & 62 & 5 & 375 & 304SS & 0.48 & 0.32 \\
\hline Hexagonal & $\mathbf{6 2}$ & $\mathbf{3}$ & $\mathbf{1 8 0}$ & $\mathbf{3 0 4 S S}$ & $\mathbf{0 . 2 5}$ & $\mathbf{0 . 1 5}$ \\
\hline Square & 250 & 5 & 250 & 304SS & 0.09 & 0.05 \\
\hline Hexagonal & 62 & 3 & 250 & 304SS & 0.311 & 0.22 \\
\hline Hexagonal & 125 & $3-5$ & 500 & 304SS & $0.26-0.29$ & 0.21 \\
\hline Hexagonal & 62 & 2.8 & 160 & Hastelloy X & 0.23 & 0.14 \\
\hline
\end{tabular}


The grid insert was designed and built to be positioned upstream from the NDCX target chamber near the longitudinal center of the final focus solenoid. The grid is shown in Fig. 3. The visible outer ring is machined from PEEK (an ultra-high vacuum-compatible organic polymer thermoplastic) with a $1.527 \mathrm{in}$. outer-diameter and $1.307 \mathrm{in.} \mathrm{inner-}$ diameter, which slightly reduces the beam pipe aperture over a length of $0.25 \mathrm{in}$. The non-magnetic stainless steel grid is positioned against a machined step in the PEEK material and held with two stainless steel fastening screws.

The installed grid is shown in Fig. 4. Two stainless steel leads were attached to the fastening screws and make electrical contact with the grid. These leads are used for mechanically adjusting the grid position during installation, and then one lead is attached to a DC voltage power supply to apply a bias potential to the grid with respect to the grounded beam pipe. A capacitive-coupling box was placed between the grid and the DC power supply, and a monitoring signal then measures the current flowing through the grid.

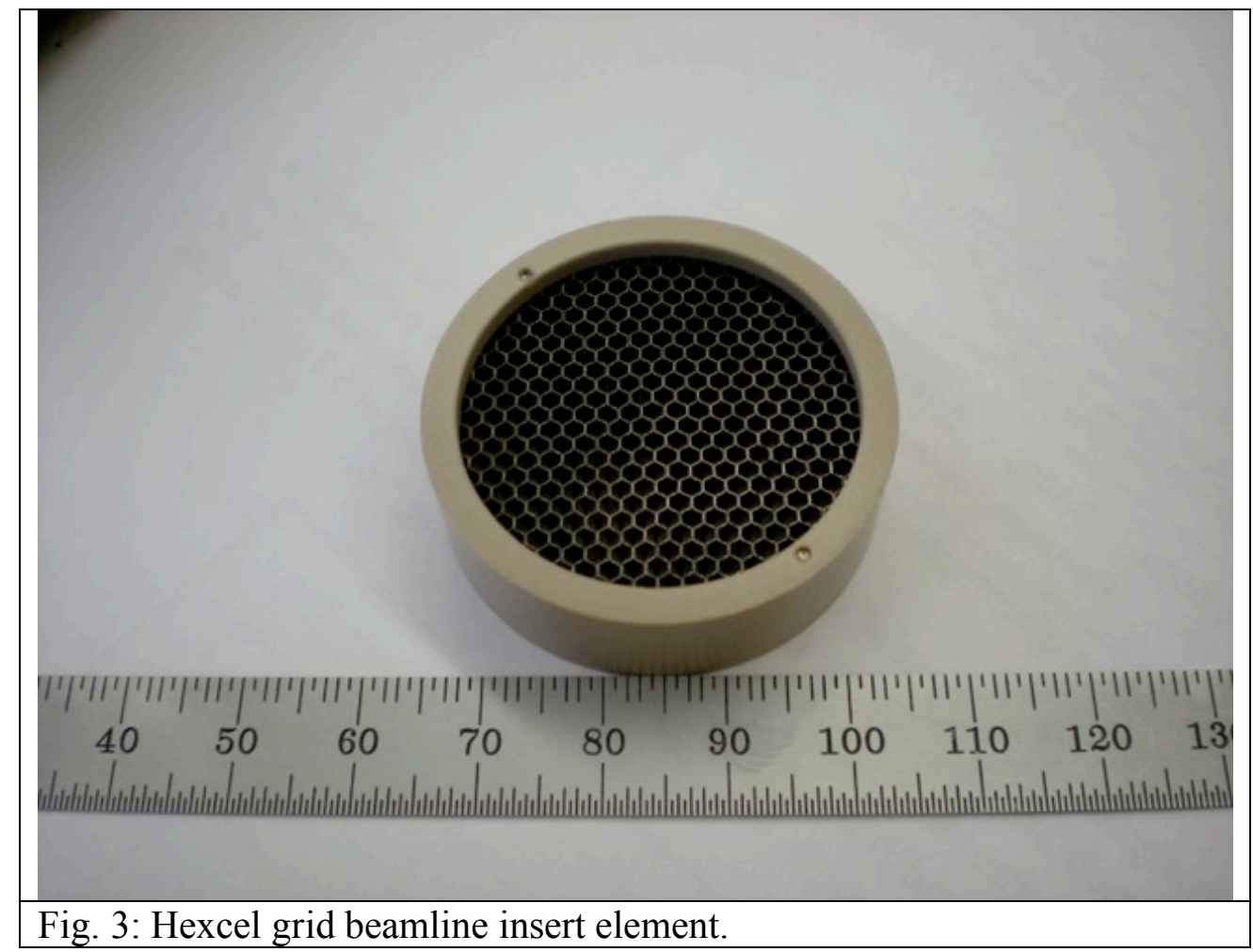




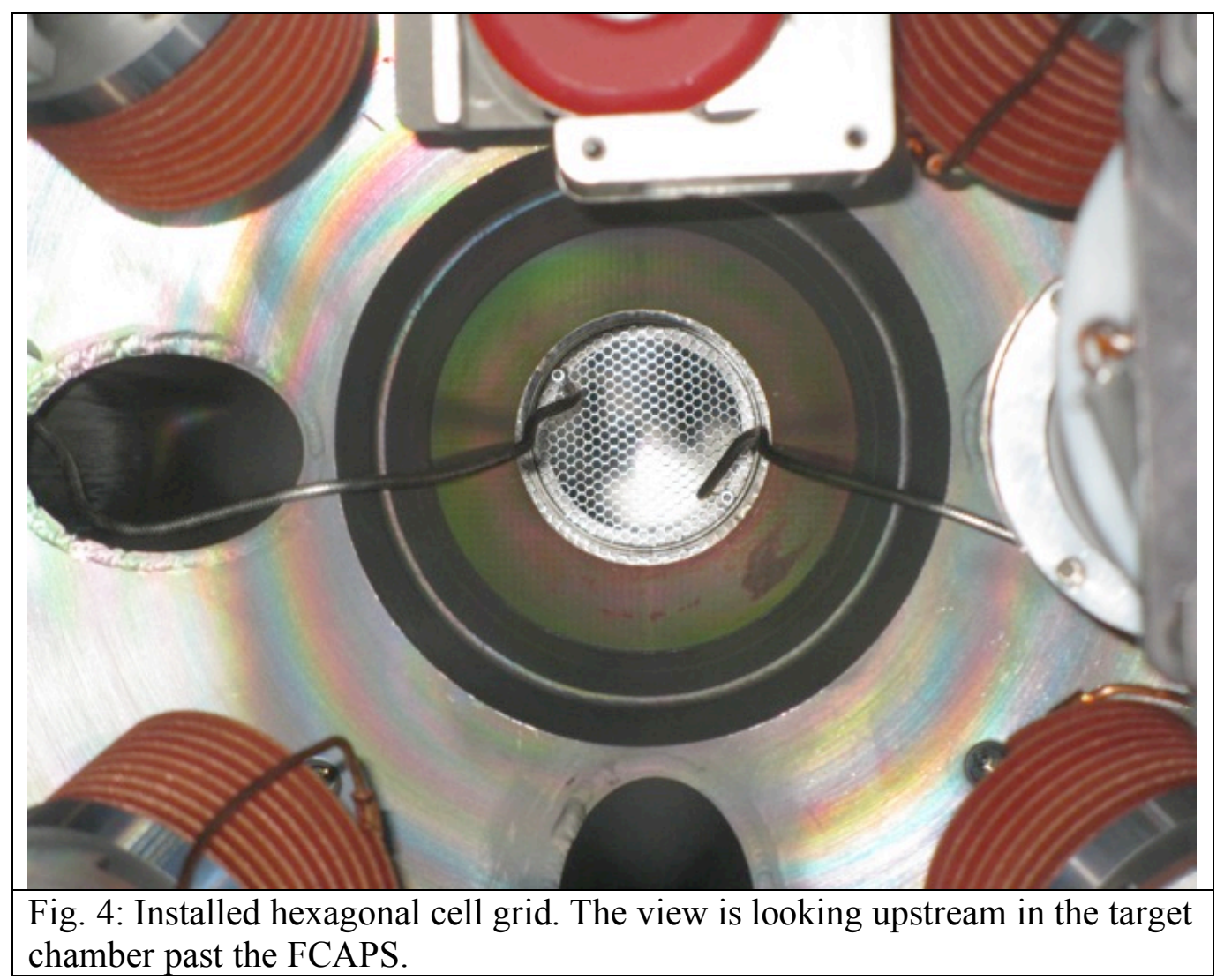

Mechanically, the hexagonal grid is a self-supporting structure and requires no additional stiffening members to maintain its geometry or position. It was, however, recognized that the eddy currents induced by the fast rise-time of the 8T final focus solenoid magnet could interact with the solenoid magnetic field and produce a $\mathrm{JxB}$ impulse that might mechanically disturb the grid structure. The grid was observed during repetitive pulsing of the magnet as the peak magnetic field was slowly increased to the maximum, 8T value. No motion of the grid was observed.

\section{Grid measurements with ion beam current}

A series of measurements determined the electrical response of the grid in the presence of the beam and any background plasma. A first series of measurements sought to quantify the beam-induced signals on the grid and any effect of bias voltage. Fig. 5 shows the bias dependence on the observed grid current as the uncompressed beam passes the grid position. In these measurements, the beam energy was $317 \mathrm{keV}$ and carried a $29.6 \mathrm{~mA}$ average current in the flat-top region. For this series, the background plasma in the target chamber was absent. While certain features of the grid monitor signals, such as the observed pulse length and rise/fall times provide a match to the beam distribution, there are other time-dependent features that are yet unexplained and require further study. 


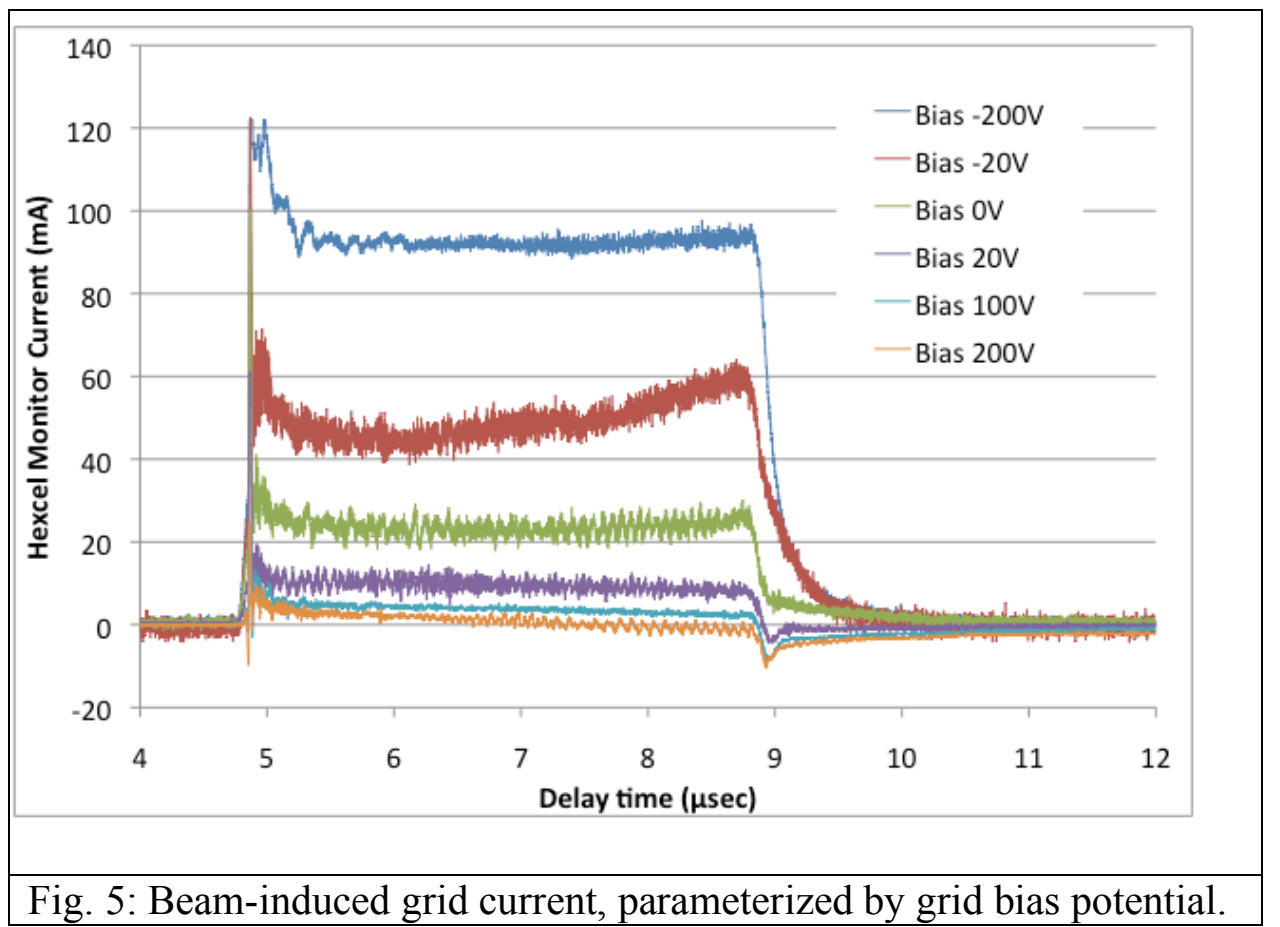

Removing the time-dependence was done by selecting the grid monitor current value at a specific delay time $(\sim 7 \mu \mathrm{sec})$. Fig. 6 shows the grid monitor current as the bias varies from $-200 \mathrm{~V}$ to $300 \mathrm{~V}$, for several beam energy and average current cases.

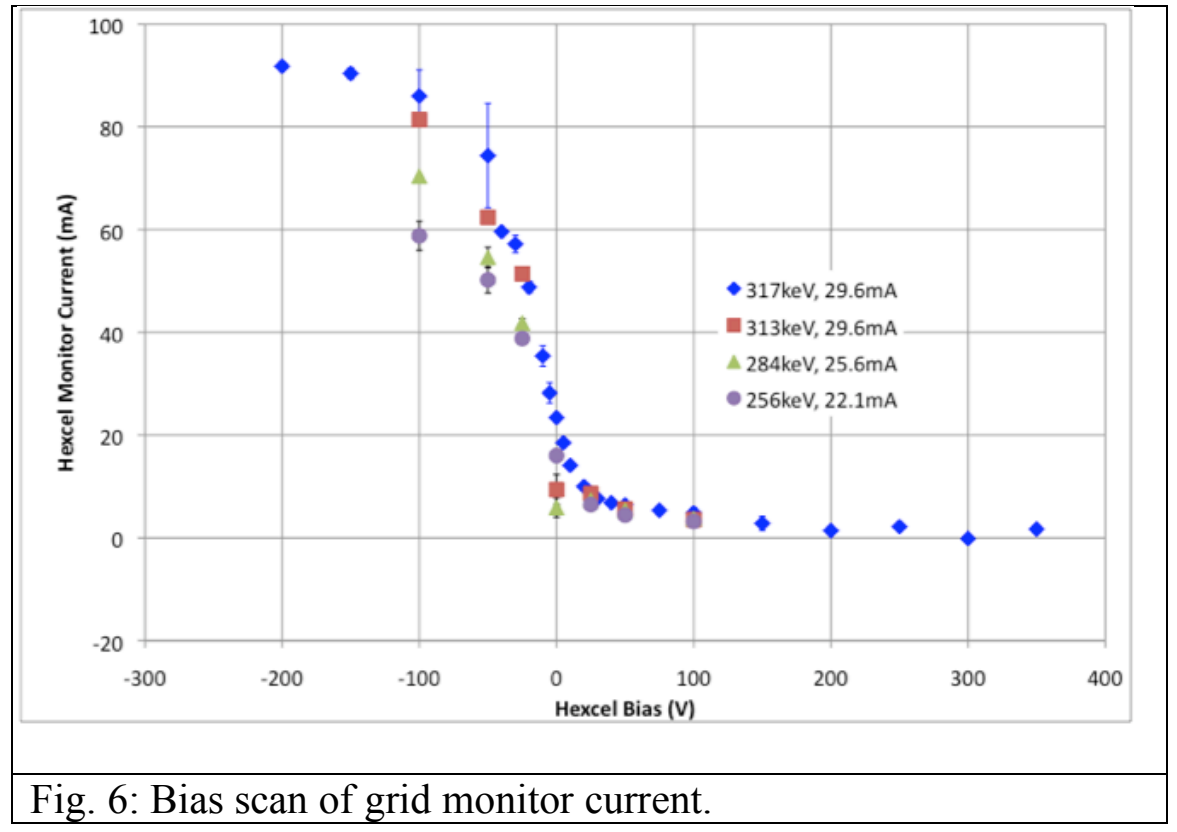

The grid monitor signal for the compressed beam is shown in Fig. 7. The beam voltage and average current in this case was again $317 \mathrm{keV}$ and $29.6 \mathrm{~mA}$, respectively. The bias 
voltage was set to $-150 \mathrm{~V}$. In this case, the background plasma from the FCAPS sources in the target chamber was present. This is evident in the background grid monitor level prior to, and following from, the beam passage. As in the uncompressed case, the beam current signal waveform is qualitatively reproduced at the grid. Further analysis would determine the instrumental resolution due to transit time through the grid (4.5 ns), capacitive induced signals, and gas ionization. Once these effects are understood, the grid might also serve as a beam current diagnostic.

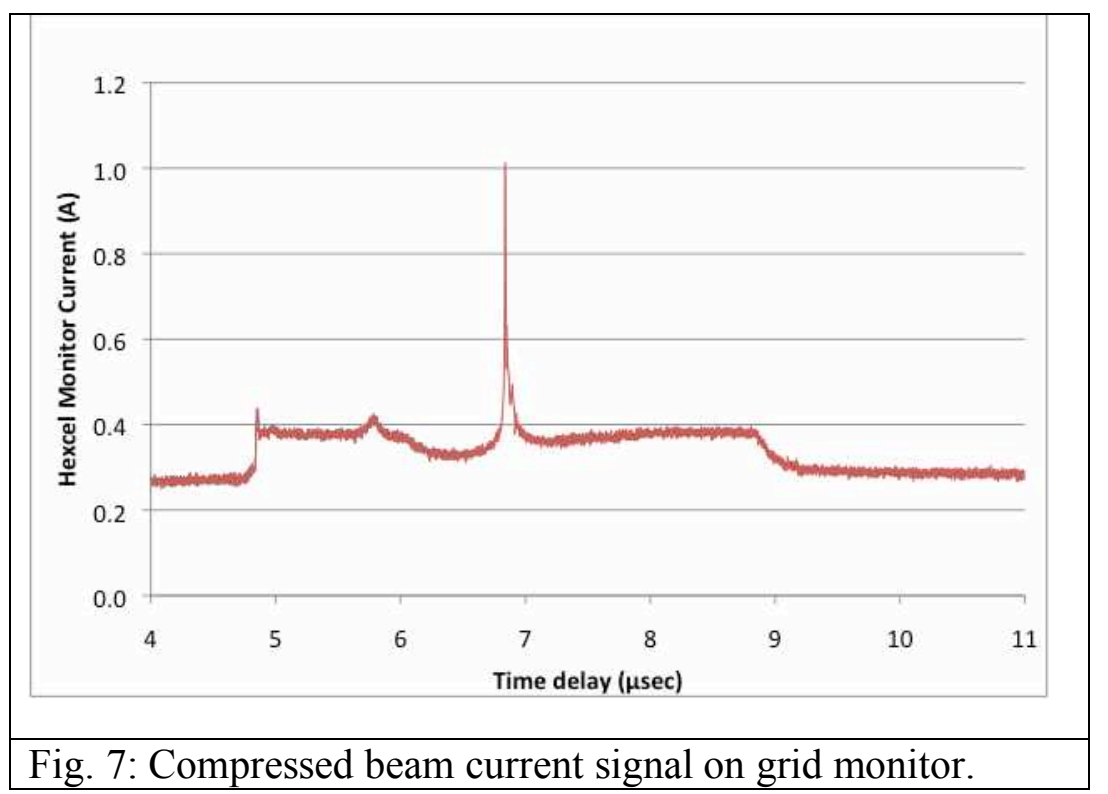

\section{Effect on the beam}

The first effect of the grid on the beam to be measured was the transmitted current to the fast Faraday cup (FFC) in the target chamber. Table 2 lists the measured FFC signal levels in the central, flat-top region of the uncompressed beam as function of beam energy. The grid was unbiased in these measurements, and the target chamber plasma was absent to avoid spurious biasing of the FFC signals. The measured beam transmission is in good agreement with the beam loss estimates previously presented in Table 1.

Table 2. Beam transmission measurements.

\begin{tabular}{|c|c|c|c|}
\hline & \multicolumn{2}{|c|}{ FFC signal (mV) } & \\
\hline Beam energy (keV) & Without grid & With grid (0V bias) & Transmission Ratio \\
\hline 284 & 30.4 & 22.2 & 0.730 \\
\hline 303 & 33.6 & 24.2 & 0.720 \\
\hline 313 & 36.4 & 25.0 & 0.687 \\
\hline 317 & 35.6 & 26.0 & 0.730 \\
\hline 324 & 36.4 & 26.8 & 0.736 \\
\hline & & Average: & $\mathbf{0 . 7 2}$ \\
\hline
\end{tabular}


Transmitted beam intensities were inferred at the target plane using a variety of measurements. The uncompressed beam current is measured upstream in the diagnostic box that precedes the induction bunching module (IBM). In the range of matching solenoid $(\{\mathrm{S} 4\})^{1}$ field strengths used, and in the presence of the neutralizing plasma column preceding the final focusing solenoid, this entire current has been measured with full transmission at the target chamber fast Faraday cup prior to the installation of the neutralizing grid. The addition of the grid reduces the transmitted current, with an assumed average transmission ratio of 0.72 . This serves to normalize the beam current and transmitted beam power impinging on the target plane and to cross-calibrate the beam current measurements with the fast Faraday cup and the total beam intensity recorded from scintillator measurements. Peak intensities are then inferred from ratios in the fast Faraday cup monitor signals, and from transverse distributions measured with the scintillator.

The uncompressed beam intensity is shown in Fig. 8 for a $324 \mathrm{keV}$ beam as a function of the upstream matching solenoid ( $\{\mathrm{S} 4\}$ ) peak field strength. A peak intensity of $\sim 100 \mathrm{~kW} / \mathrm{cm}^{2}$ is indicated at a field strength of $1.75 \mathrm{~T}$ for zero bias voltage on the grid.

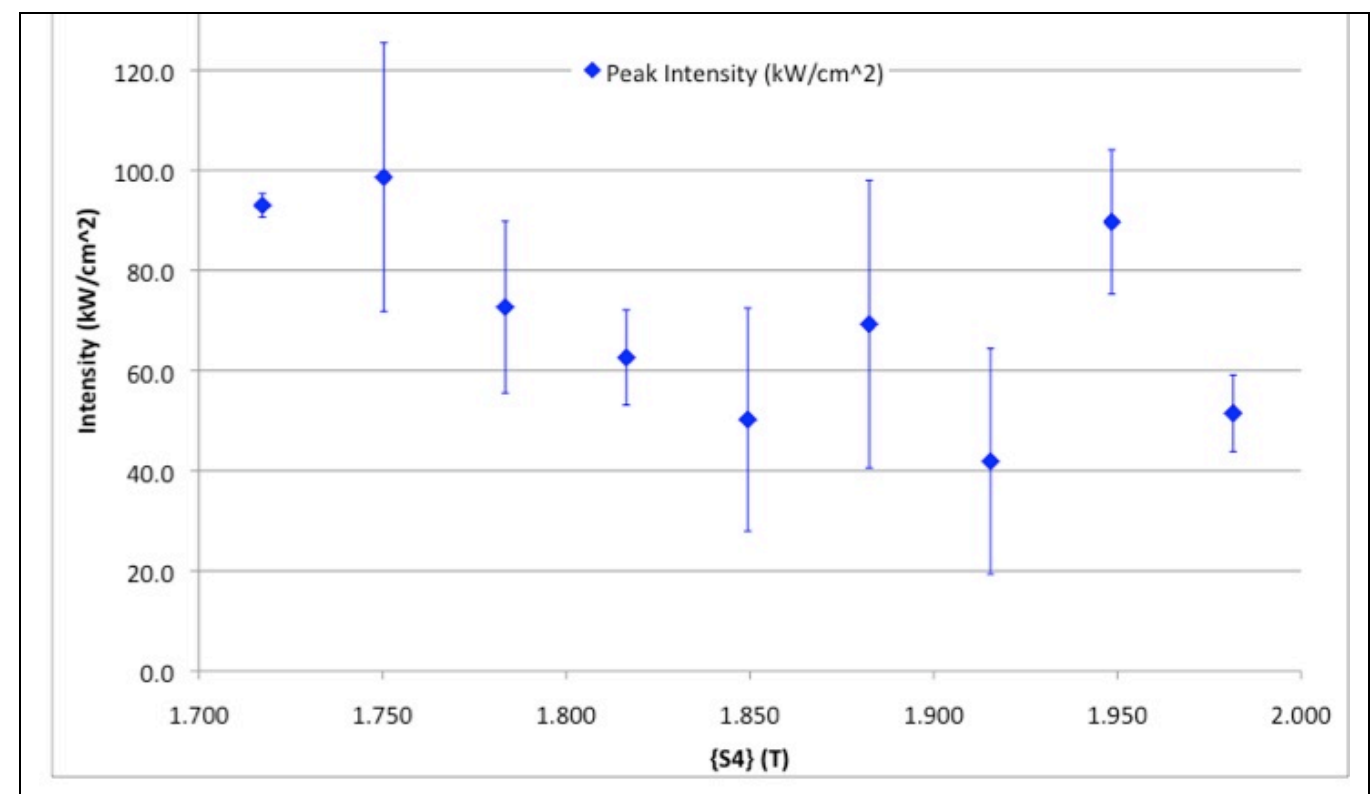

Fig. 8: Uncompressed beam intensity. 0V bias, $324 \mathrm{keV}$

The compressed beam power and pulse energy is shown in Fig. 9. The $324 \mathrm{keV}$ carrying $30 \mathrm{~mA}$ of average (uncompressed) current delivers a beam power of $\sim 7 \mathrm{~kW}$, accounting for the $72 \%$ beam transmission through to the target plane. The peak power in the compressed pulse delivered to the target plane was measured at $\sim 470 \mathrm{~kW}$, with $\sim 1.5 \mathrm{~mJ}$ in the $\sim 5 \mathrm{~ns}$ (FWHM) compressed pulse.

\footnotetext{
${ }^{1}$ The \{\} braces denote that the magnetic field is scaled proportional to $\mathrm{E}_{\mathrm{b}}{ }^{1 / 2}$ to keep the rms beam envelope in the matching solenoids and un-neutralized drift sections unchanged for a space charge dominated beam.
} 


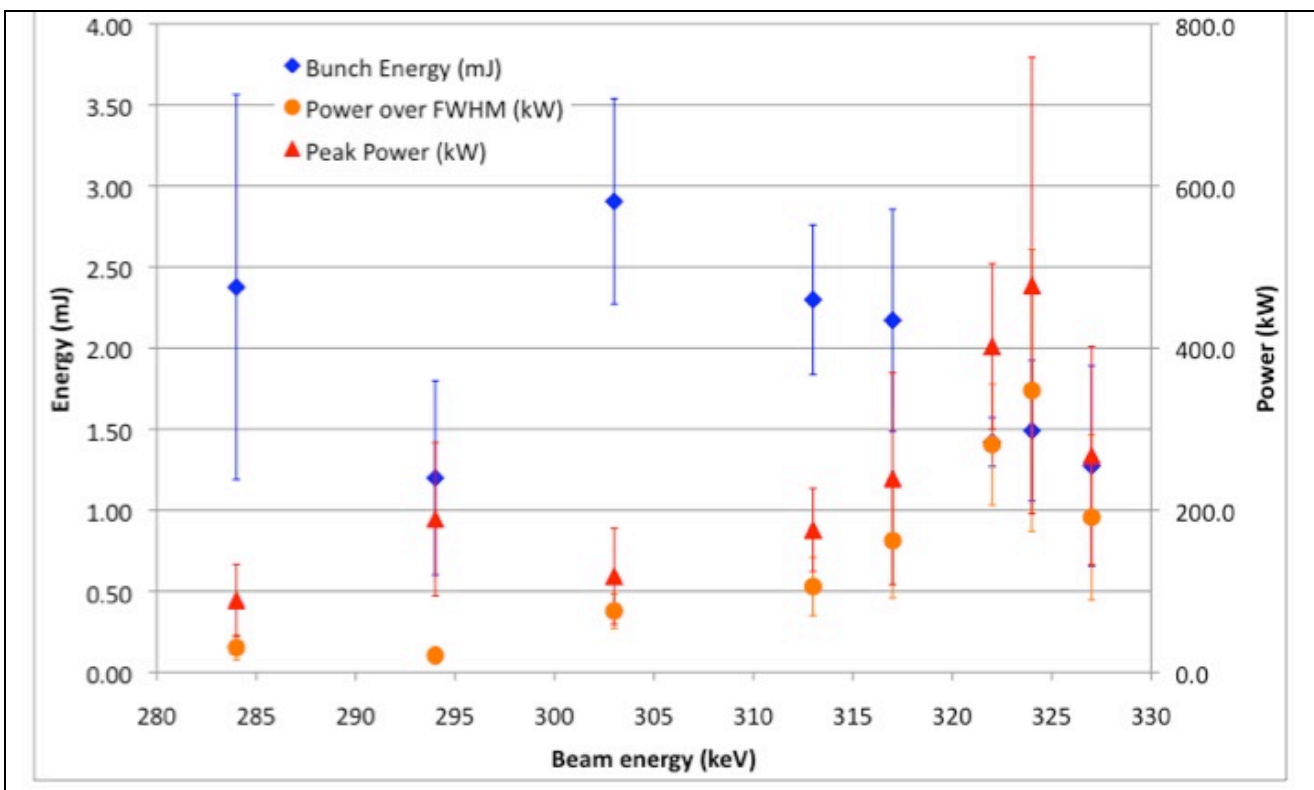

Fig. 9: Compressed beam power and energy

The peak fluence and intensity of the compressed bunch region of the beam pulse is shown in Fig. 10. The matching solenoid tune for the peak compressed beam tune differs from that of the uncompressed beam (compare with Fig. 8). The peak intensity measured was $\sim 6 \mathrm{MW} / \mathrm{cm}^{2}$ and the peak fluence of the compressed bunch was $\sim 18 \mathrm{~mJ} / \mathrm{cm}^{2}$.

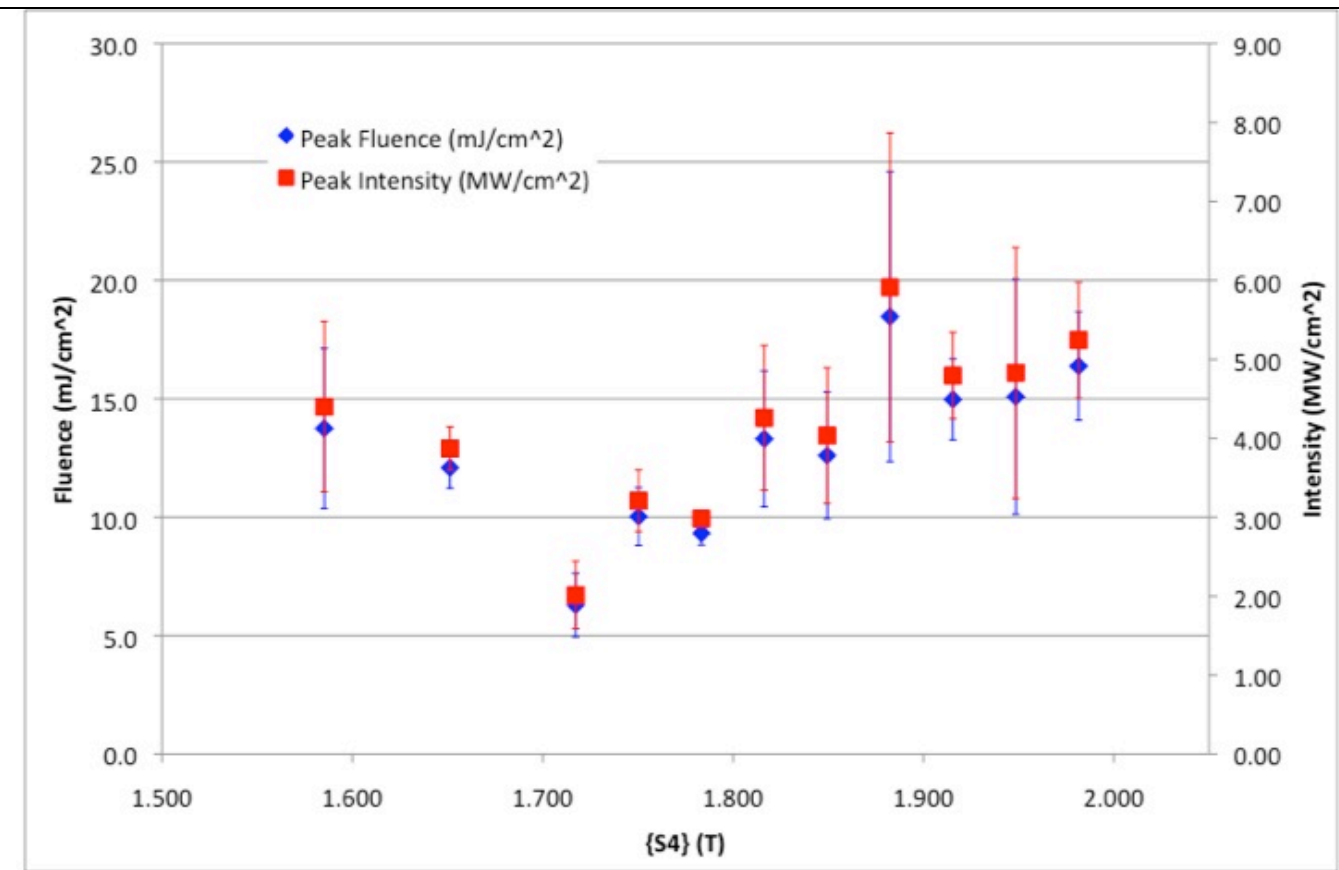

Fig. 10: Compressed beam fluence and intensity. 
A bias scan was performed to examine any measurable influence of the grid bias voltage on the beam's uncompressed and compressed pulse peak intensity. The results show (Fig. 11) that a $2 \mathrm{X}$ variation in both quantities over the bias potential range of $-200 \mathrm{~V}$ to $200 \mathrm{~V}$. The relatively coarse resolution in the bias scan $(50 \mathrm{~V})$ may be obscuring subtle effects in the $-20 \mathrm{~V}$ to $20 \mathrm{~V}$ range. This will be examined in further detail.

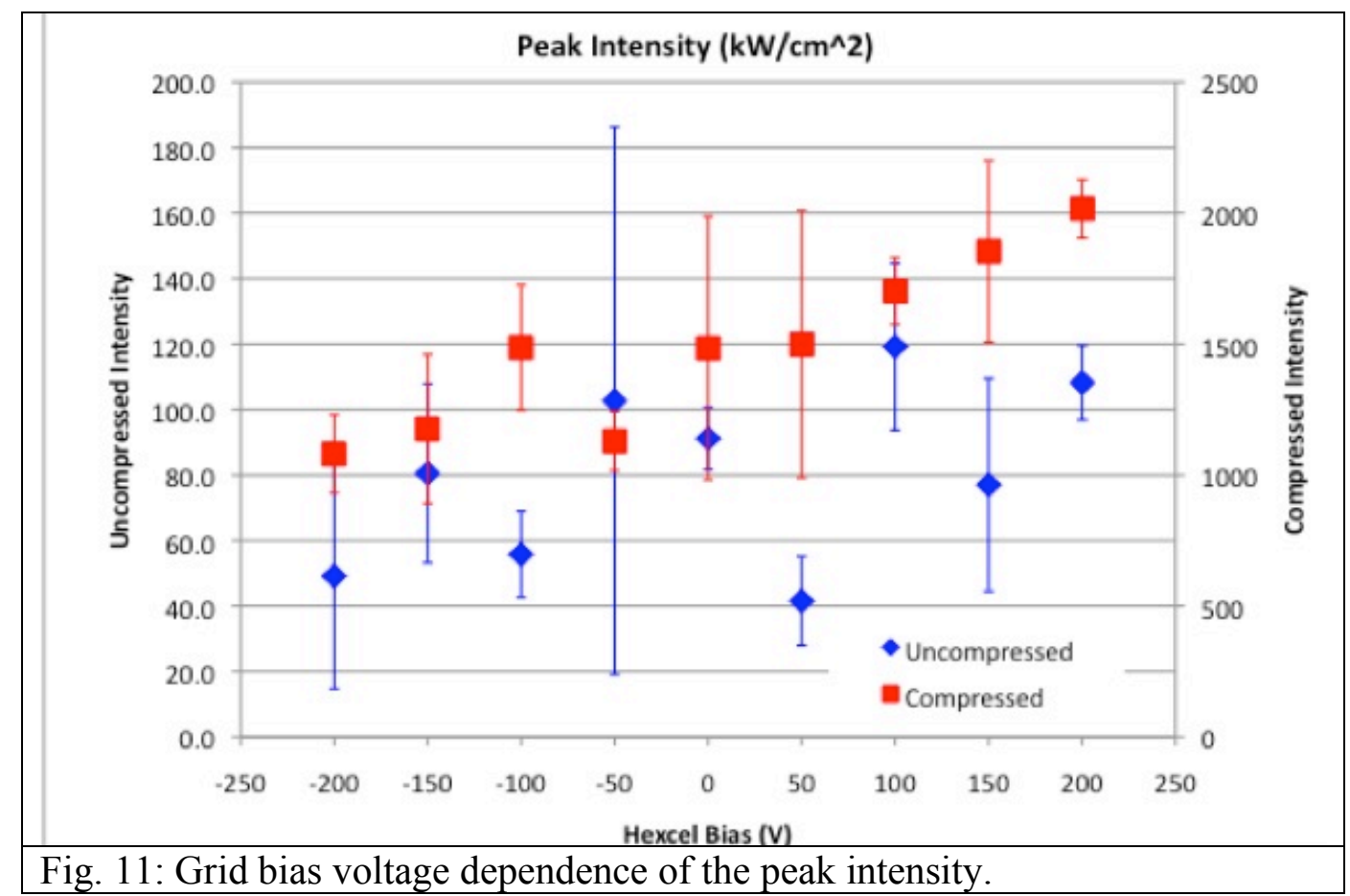

\section{Compact Ferroelectric Plasma Source (FEPS)}

A compact ferroelectric plasma source that is based on the technology used in the existing 3" diameter, 2-m-long ferroelectric plasma source has been tested. The compact ferroelectric plasma source is a 1.5 " long, 1.125" inner-diameter, 1.5" outer-diameter barium titanate ring that has a grounded, perforated steel sheet inner electrode, and a silver paint outer electrode. A high-voltage pulse applied to the outer surface creates a large surface charge density on the inner surface due to the high dielectric coefficient of the barium titanate. The resulting large electric field causes plasma formation (see Fig. 12). 


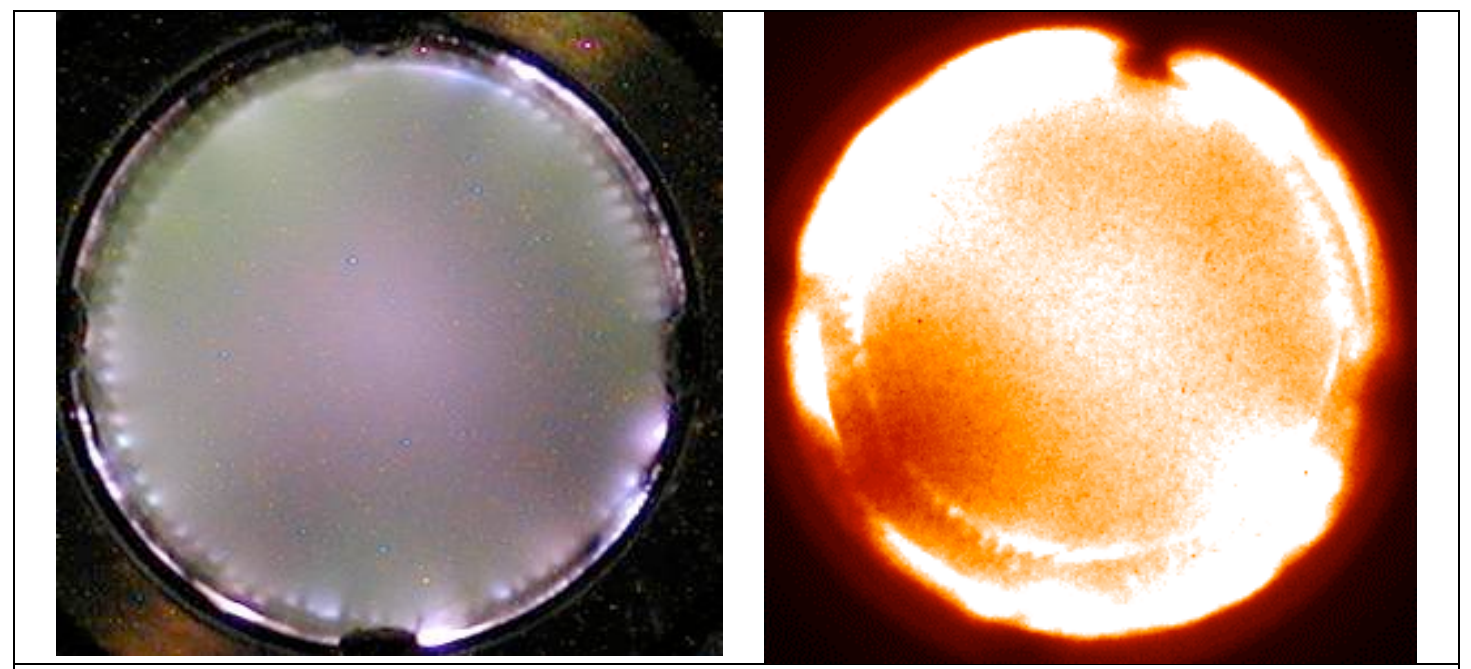

Fig. 12: An optical image with a commercial digital camera (left) clearly shows the plasma being emitted from the holes in the perforated steel sheet inner electrode. A false-color image from a scientific-grade intensified CCD camera system (right) allows for quantitative analysis of the emitted light.

The plasma density in the compact ferroelectric plasma source is expected to be greater than in the 3" diameter plasma source because the volume filled by plasma scales with the radius-squared, while the amount of plasma created scales with the surface area of the cylinder, which scales with the radius. Further, being a single 1.5" long cylinder, issues with flashover protection between adjacent ceramic rings are avoided, allowing for the possibility of driving the plasma source at higher voltages to produce more plasma. An IGBT-switched $0.2 \mu \mathrm{F}$ capacitor charged to $8 \mathrm{kV}$ drives a peak current of over $200 \mathrm{~A}$ through the plasma source for just over $2 \mu$ s. Langmuir probe measurements of the plasma density at the center of the device show that the density is approximately $5 \times 10^{11}$ $\mathrm{cm}^{-3}$, which is an order of magnitude greater than the density obtained with a similar charging voltage in the 3" diameter ferroelectric plasma source. This increase is greater than the contribution from the geometric scaling, and may be due to field enhancement near the inner surface or other details of the modified design. Figure 2 shows a $1 \mu \mathrm{s}$ exposure taken $4 \mu \mathrm{s}$ after the plasma formation begins. The interior volume has been filled by plasma and there is still plasma being generated at the inner surface. An examination of a slice of the image data through the center of the image shows that the plasma density is likely spatially uniform. 


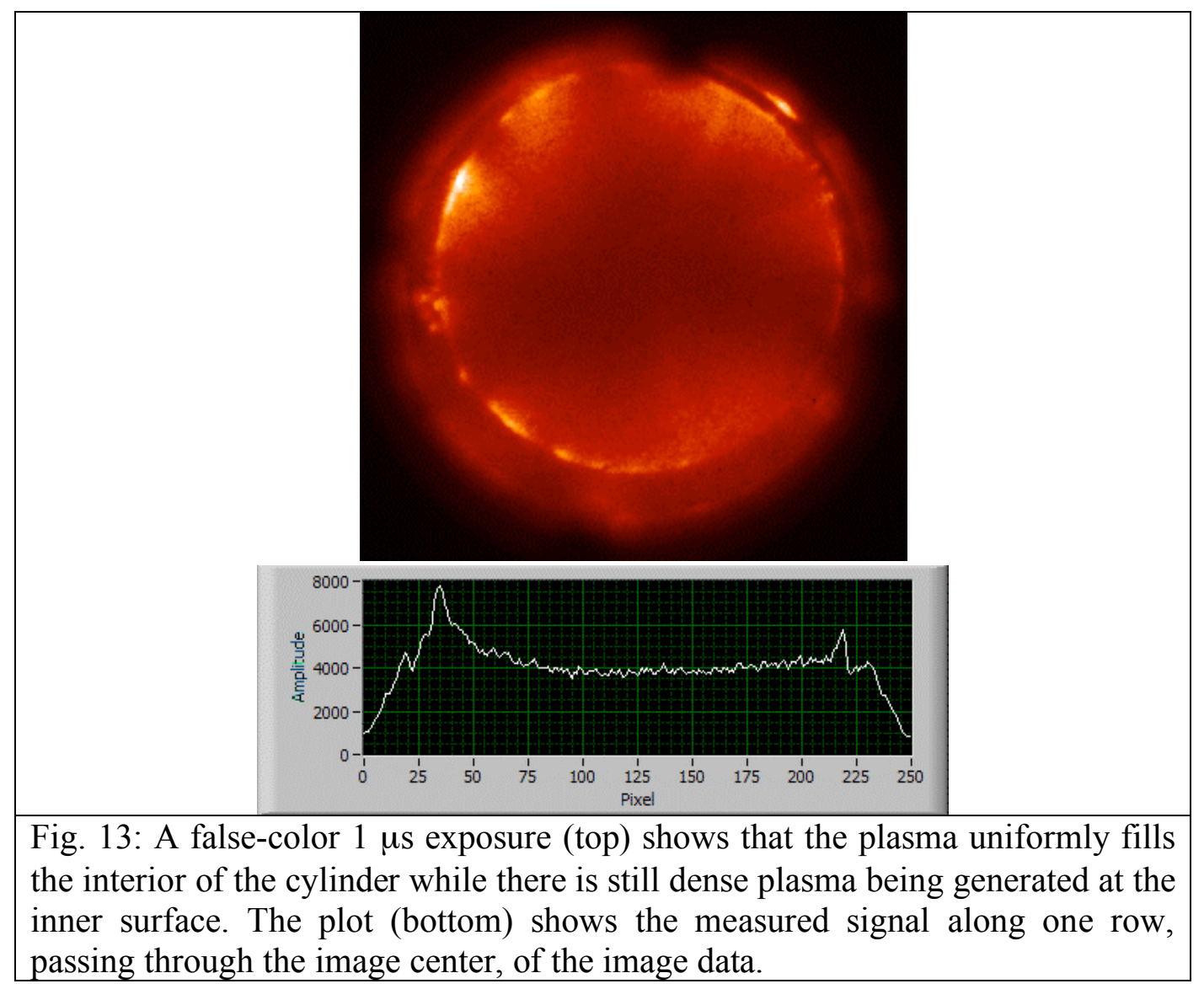

Even without further increases in plasma density, the compact ferroelectric plasma source is a candidate for use within the pipe of the NDCX-I Final Focus Solenoid (FFS) in order to generate plasma on magnetic field lines that connect to points at large radius at the FFS midplane. Tests have been performed at PPPL with the 1.5" outer-diameter plasma source and a custom-machined grounded pipe that fits over the plasma source and provides a $2 \mathrm{~mm}$ annular gap between the outer surface of the plasma source and the pipe. This is a more closed geometry than the $8 \mathrm{kV}$ tests described above, and closely resembles the NDCX1 setup (without the magnetic field). The high-voltage lead has been modified to pass, axially, through the end-cap of the plasma source and Kapton sheet insulation has been added to the outer surface to suppress arcing. The plasma source has been successfully operated at $5 \mathrm{kV}$ charging voltage, where the plasma density is $1 \times 10^{11}$ $\mathrm{cm}^{-3}$. The voltage is limited by concerns of possible breakdown at higher voltages near the high current leads. In preparation for testing at LBNL with a multi-Tesla magnet, 1.5"-long barium titanate cylinders with a 1.375" outer-diameter and a 1.0" innerdiameter have been ordered that will fit within the FFS pipe while allowing a $2 \mathrm{~mm}$ gap between the plasma source and the grounded pipe wall. 


\section{Reducing the gap between the FEPS and the Final Focus Solenoid}

A modification to the NDCX neutralized transport was implemented to increase the local plasma density in the terminal region near the target chamber, where the local beam density is increasing rapidly. To that end, the isolation gate valve (Fig. 1) located between the exit of the FEPS transport section and the final focus solenoid was removed, and another isolation gate valve was installed approximately one meter upstream. The modified beamline is depicted in Fig. 14. This shortened the unneutralized drift length between the FEPS and the final focus solenoid from $12 \mathrm{~cm}$ to $5 \mathrm{~cm}$.

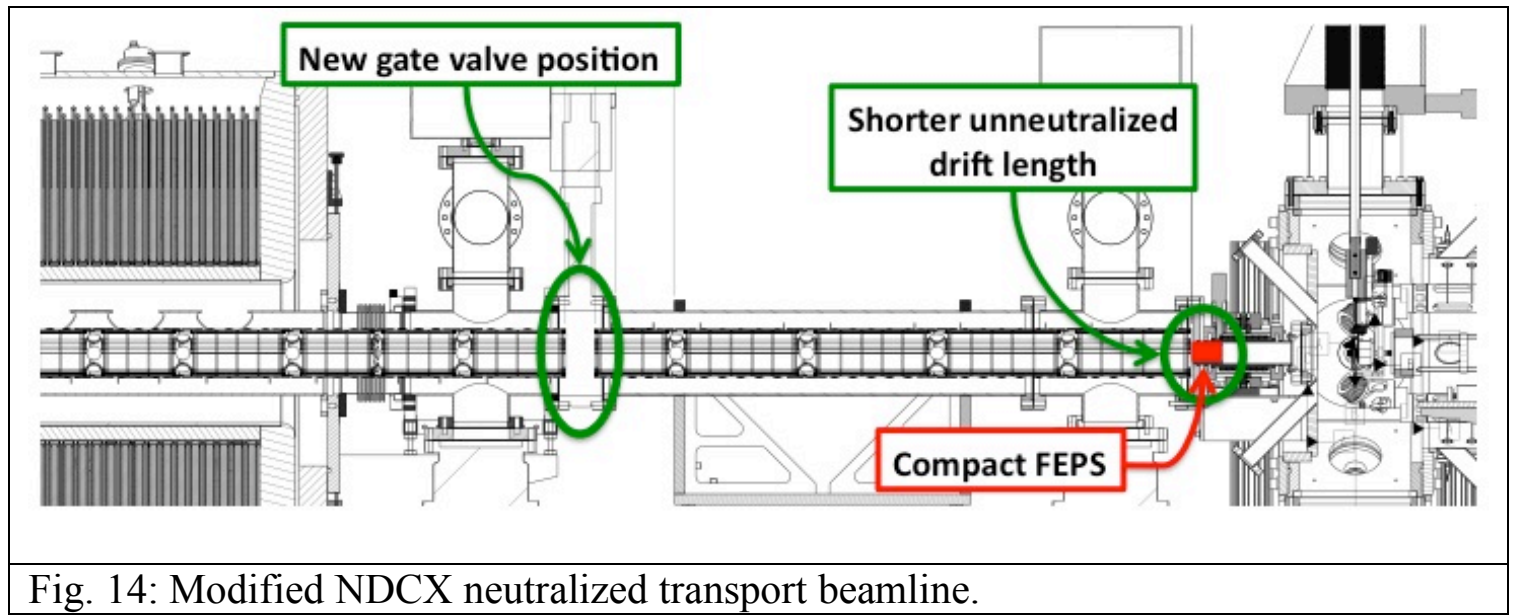

We have performed WARP simulation studies of the two beamline geometries with varying degrees of neutralizing plasma fill in the region from the FEPS exit to the target. The attached plot in Fig. 15 compares the energy fluence on target for four cases. The black shows the case with plasma everywhere. The other three show cases that have a plasma extending radially to $5 \mathrm{~mm}$ in the solenoid, and varying gap length. The cases with $0 \mathrm{~cm}$ and $5 \mathrm{~cm}$ gap length are the same to within the statistical simulation noise. The $12 \mathrm{~cm}$ gap case shows about half of the fluence at target center, and about a factor two larger spot size. 


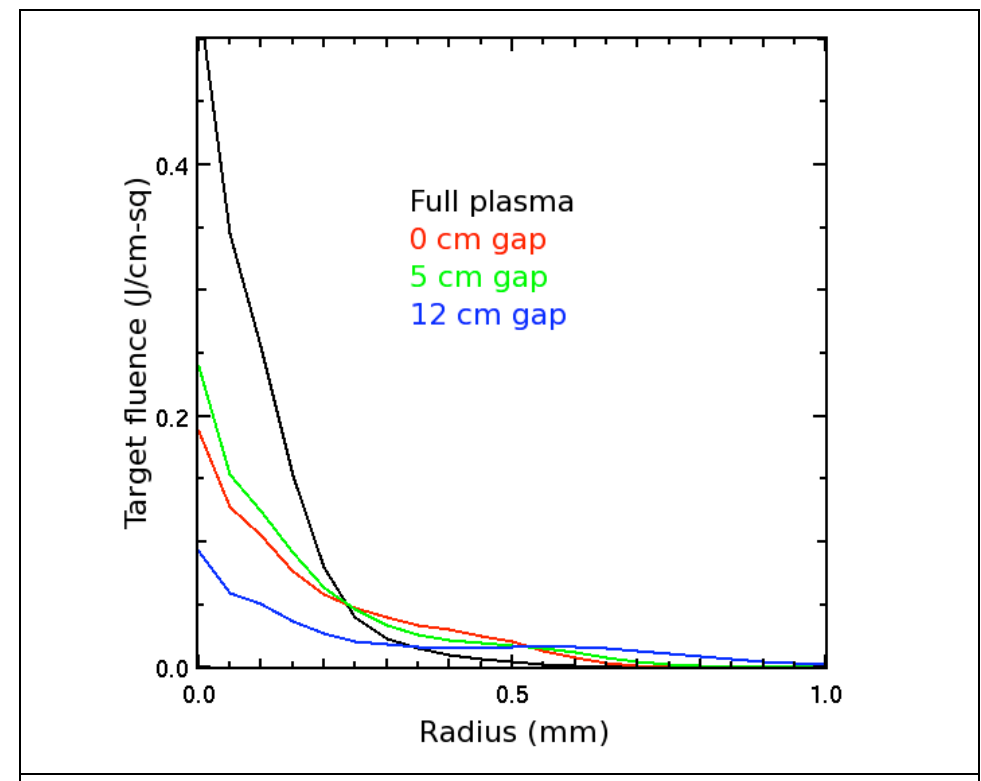

Fig. 15: Target-plane beam fluence simulation results.

\section{Summary and Conclusions}

We have presented measurements and simulations of beam intensities and compressed pulse fluences on the target plane with a modified NDCX beamline to provide more complete coverage and higher densities of neutralizing plasma. Early and contemporaneous simulations indicate a $2 \mathrm{X}$ improvement in compressed beam pulse fluence with a shorter longitudinal gap between the FEPS exit and the final focus solenoid.

We have installed a biased hexagonal grid at the center of the final focus solenoid to provide greater coverage of neutralizing electrons at larger radii than can be filled with the target chamber FCAPS plasma when the solenoid is operating. Measurements are continuing, but indicate a subtle bias dependence on the target plane beam fluence that may also significantly increase compressed pulse intensity and fluence on target.

We have produced a design and working prototype of a compact FEPS source that will be installed in the beamline between the FEPS exit and extending into the upstream end of the final focus solenoid.

\section{References}

[1] S.A. MacLaren, et. al., Phys. Plasmas 9, 1712 (2002).

[2] G.A. Krafft, "Collective Focusing of Intense Nonrelativistic Ion Beams by CoMoving Electrons", Ph.D. Thesis (1987), LBL-21523.

[3] A.W. Molvik, et. al, Phys. Rev. ST Accel. Beams 7, 093202 (2004).

[4] http://www.hexcel.com/Products/Core+Materials/Honeycomb 